\title{
Molecular Imaging Biomarkers for Oncology Clinical Trials
}

\author{
David A. Mankoff ${ }^{1}$, Daniel A. Pryma ${ }^{1}$, and Amy S. Clark ${ }^{2}$ \\ ${ }^{1}$ Radiology/Nuclear Medicine, Perelman School of Medicine, University of Pennsylvania, Philadelphia, Pennsylvania; \\ and ${ }^{2}$ Medicine/Medical Oncology and Abramson Cancer Center, Perelman School of Medicine, University of Pennsylvania, \\ Philadelphia, Pennsylvania
}

\begin{abstract}
Biomarkers can be used to characterize disease status or predict disease behavior. Cancer biomarkers have typically relied on assays of blood or tissue; however, molecular imaging has a promising and complementary role as a cancer biomarker. This "Focus on Molecular Imaging" article reviews the current role of biomarkers to direct cancer clinical trials and clinical practice, along with current and future cancer biomarker applications of molecular imaging.
\end{abstract}

Key Words: cancer biomarkers; clinical trials; cancer therapy

J Nucl Med 2014; 55:525-528

DOI: 10.2967/jnumed.113.126128

The term biomarker is used to describe a biologic measure that characterizes disease status or predicts disease behavior (1). With the goal of increasingly individualized cancer treatment, biomarkers have become an increasingly important part of cancer care (1). Biomarkers can be used to detect cancer; for example, prostate-specific antigen is used for prostate cancer screening. Cancer biomarkers can also be used to direct cancer therapy; for example, an assay of breast cancer estrogen receptor (ER) expression is recommended for all patients with invasive breast cancer to determine the need for endocrine therapy (2). In this article, we will review the use of molecular imaging as a biomarker to direct cancer therapy for clinical trials and eventual clinical practice, focusing largely on breast cancer, for which individualized therapy and cancer biomarkers have become an important part of clinical trials and clinical practice $(1,2)$.

Leaders in biomarker research have defined essential features for cancer biomarker research that include guidelines for the validation and use of biomarkers in cancer clinical trials and clinical care $(1,3)$. The REMARK guidelines (Reporting Recommendations for Tumor Marker Prognostic Studies) are the most widely accepted reporting criteria for cancer biomarker trials (3). Important features of these guidelines and biomarker trial reporting include the following $(1,3)$ : determination of the analytic validity of the biomarker, including calibration, precision, and accuracy of the measurement; the intended use for directing cancer therapy; the magnitude of the biomarker's clinical value; and the quality of the clinical trial data

Received Dec. 12, 2013; revision accepted Feb. 24, 2014.

For correspondence or reprints contact: David A. Mankoff, 116 Donner Building, Hospital of the University of Pennsylvania, 3400 Spruce St., Philadelphia, PA 19104-4283.

E-mail: david.mankoff@uphs.upenn.edu

Published online Mar. 10, 2014.

COPYRIGHT (c) 2014 by the Society of Nuclear Medicine and Molecular Imaging, Inc. supporting the biomarker. The analytic validity of the marker includes the technical ability to perform the measurement, the threshold for considering a biomarker positive or negative, and the reproducibility of the measurement. The magnitude of the biomarker predictive value and the quality of clinical trial data supporting biomarker use are key factors in the clinical acceptance of the biomarker. The strongest level of evidence (level 1) supporting a biomarker comes from prospective clinical trials specifically designed to test the impact of the biomarker on patient outcome when used to direct treatment $(1,3,4)$. Achieving this level can be challenging for imaging biomarkers, because clinical trials can be expensive and may not provide benefit for the patient. An alternative approach is to test the biomarker in a purely observational study with prospectively defined biomarker endpoints, considered level 2 evidence $(3,4)$; for example, studies of breast cancer multigene assays can be applied to data from randomized controlled adjuvant therapy trials to validate their value in predicting the need for chemotherapy to prevent recurrence (5).

\section{INTEGRATED AND INTEGRAL BIOMARKERS}

The terms integral and integrated are increasingly used to describe the role of a biomarker in a clinical trial (Fig. 1) $(3,6)$. An integral biomarker is essential to the clinical trial. Integral biomarkers directly affect clinical trial procedures, such as to direct stratification between arms of a treatment study. Integral biomarkers are typically biomarkers for which the analytic method has been extensively validated in prior trials. The inclusion of an integral biomarker in a clinical trial can provide level 1 evidence supporting the biomarker for the specific indication tested in the trial. For example, interim ${ }^{18} \mathrm{~F}$-FDG PET/CT early in the course of lymphoma treatment can be used as an integral biomarker to direct subsequent treatment, a common feature in recent lymphoma therapy trials (7). When clinical trials with an integral biomarker prove that a particular drug or drug combination is effective, the biomarker often becomes part of the drug indication for use in clinical practice. For example, in trials of ${ }^{223} \mathrm{Ra}$ for treatment of bone-dominant castrate-resistant prostate cancer, evidence of osteoblastic bone metastases by bone scanning was required for entry into the trial; that is, bone scanning was an integral imaging biomarker. The clinical indication for ${ }^{223} \mathrm{Ra}$ recently approved by the U.S. Food and Drug Administration requires evidence of osteoblastic lesions by bone scintigraphy $(8)$.

Integrated biomarkers, on the other hand, are markers that are measured in the context of a prospective trial but are not used to direct treatment. An example is the recently published imaging result for the NeoALTTO trial (Neoadjuvant Lapatinib and/or Trastuzumab Treatment Optimization) (9), in which serial ${ }^{18}$ F-FDG PET/CT was 


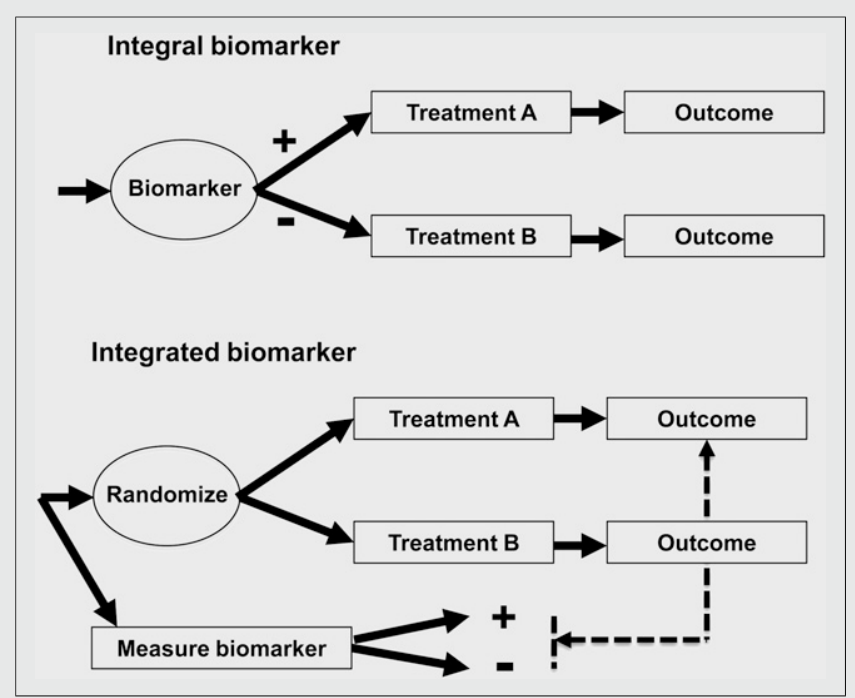

FIGURE 1. Diagram illustrating integral and integrated biomarkers. Integral biomarker is essential to the trial and is typically used to select patients or direct therapy. Integrated biomarker is included as a key observation in the trial but is not used to direct the trial. Typically, only well-validated biomarkers are used as integral biomarkers. Trials with integrated biomarkers are often designed to test utility of biomarker as predictive measure.

performed in the setting of a trial comparing the efficacy of different HER2-directed treatments in breast cancer with the goal of testing ${ }^{18} \mathrm{~F}-\mathrm{FDG}$ PET/CT as an early marker of drug efficacy. Once validated as an integrated biomarker, the biomarker can then go on to be used as an integral biomarker in subsequent clinical trials.

\section{BIOMARKERS FOR CANCER THERAPY}

Biomarkers for cancer therapy generally fall into one of several categories:

\section{Predictive and Prognostic Markers}

Predictive markers indicate the aggressiveness of a particular patient's cancer, the likely course of disease, or the likelihood of response to treatment. Prognostic markers are a class of predictive markers that assess the aggressiveness of a particular cancer and the likelihood it will lead to death. In principle, prognostic measures are intrinsic to the patient and the cancer and are independent of treatment. On the other hand, therapeutic predictive markers describe the likelihood of response to specific treatments and can be used to select treatment most likely to be effective for a particular patient's cancer. In practice, markers may have both prognostic and predictive features. An example is ER expression in breast cancer. ER expression is both a prognostic and a predictive marker. ER expression predicts a less aggressive tumor and more favorable disease course compared with non-ER-expressing tumors. In addition, a lack of ER expression predicts that breast cancer response to endocrine therapy is unlikely (2). More recently, the use of multigene assays such as Oncotype DX (Genomic Health Inc.) has helped predict tumor behavior and therapeutic response. Such assays are commonly used to determine the need for aggressive treatment such as adjuvant chemotherapy (5).

\section{Early-Response Markers}

Early-response markers, sometimes referred to as pharmacodynamic markers, indicate that there has been a biologic response to treatment, namely that the chosen cancer drug has produced an effect on the tumor. The lack of a pharmacodynamic effect indicates that the drug is unlikely to work. Identifying a lack of response, and therefore therapeutic futility, may be the most important use of early-response indicators. An example of a tissue-based early-response indicator is serial assay of tumor proliferation, such as using $\mathrm{Ki}-67$ immunohistochemistry to assess early response to breast cancer endocrine therapy (10).

\section{Surrogate Endpoints}

A surrogate endpoint is a marker of therapeutic success that is strongly associated with patient outcomes such as disease-free survival, progression-free survival, or overall survival. An example of a tissue surrogate endpoint is complete pathologic response of breast cancer to presurgical (neoadjuvant) systemic therapy, which has been accepted by the U.S. Food and Drug Administration as a surrogate endpoint for efficacy of breast cancer treatment (11).

\section{EVOLVING USE OF BIOMARKERS IN CLINICAL TRIALS}

Thus far in cancer care, the biomarkers used in clinical practice have been almost entirely based on in vitro assays of tissue or blood samples (3). Molecular imaging biomarkers offer several distinct features that are highly complementary to methods based on tissue sampling (6). Imaging is noninvasive and therefore better suited to serial assay. This is especially important in imaging specific drug pharmacodynamics and early tumor response. In addition, imaging surveys the entire patient and can therefore assess the entire disease burden. Imaging can measure biomarker expression at all disease sites and avoids the sampling error that occurs in tissue sample assays, especially when there is significant tumor heterogeneity. There are, however, some disadvantages to imaging biomarkers versus tissue biomarkers. Although samplebased methods can assay several different processes at once, such as the expression of a large number of different genes, imaging can typically sample at most 1 or 2 processes simultaneously. Furthermore, whereas it is possible to batch tissue samples for assay at a central facility, imaging needs to be performed on one subject at a time. The need for sophisticated equipment and imaging probes contributes to the cost of imaging biomarkers and can limit the number of subjects who can undergo imaging in a clinical trial. Conversely, if an imaging biomarker could reduce the needed sample size or trial duration, its use could actually decrease the cost of a trial. Overall, tissue and imaging biomarkers have highly complementary capabilities that can lead to considerable synergy in clinical trials and clinical practice.

\section{Molecular Imaging as a Predictive Marker}

Perhaps the clearest example of molecular imaging as a predictive biomarker is PET imaging of ER expression, most widely performed using ${ }^{18} \mathrm{~F}$-fluoroestradiol. ${ }^{18} \mathrm{~F}$-fluoroestradiol uptake has been shown to correlate with tissue-based assays of ER expression (12). As with tissue-based ER assays, ${ }^{18} \mathrm{~F}$-fluoroestradiol PET is predictive of breast cancer endocrine responsiveness, and importantly, a lack of ${ }^{18} \mathrm{~F}$-fluoroestradiol uptake strongly predicts a lack of response. Early trials on other ER-expressing cancers such as endometrial cancer also support a potential predictive role (13). Through the support of the National Cancer Institute (NCI) Cancer Imaging Program, there is now an NCI-held investigational new drug application (IND) for ${ }^{18} \mathrm{~F}$-fluoroestradiol to support clinical trials, and there has been successful completion of a small prospective phase 2 trial (Fig. 2) (14). This approach may 
be helpful for trials of novel endocrine therapies for breast cancer, especially to help select patients whose tumor expresses the therapeutic target (ER); however, more rigorous, carefully controlled, and properly powered studies to establish the negative predictive value of the absence of ${ }^{18} \mathrm{~F}$-fluoroestradiol uptake with well-defined uptake thresholds are needed to support this indication. Multicenter trials performed under the NCI IND that can address this need are being planned. Several other molecular imaging approaches for measuring regional expression of therapeutic cancer targets are also emerging, including progesterone receptor, HER2, and epidermal growth factor receptor (15).

\section{Molecular Imaging as an Early-Response Indicator}

There are several studies showing the potential of ${ }^{18} \mathrm{~F}-\mathrm{FDG}$ PET as an early indicator of response to therapy for breast and other cancers. Studies have shown that, on average, a decline in ${ }^{18} \mathrm{~F}$-FDG uptake early in the course of chemotherapy is predictive of subsequent response and patient survival, although the ability to identify nonresponders is limited by overlap in ${ }^{18} \mathrm{~F}$-FDG uptake levels between different categories of response in many trials $(7,16)$. Early posttherapy ${ }^{18}$ F-FDG PET is increasingly used in therapeutic clinical trials as an early-response indicator, including use as an integral biomarker to direct therapy selection in ongoing lymphoma trials (7). ${ }^{18} \mathrm{~F}$-FDG has also been shown to be predictive of breast cancer response to endocrine therapy, indicated by either an increase in ${ }^{18} \mathrm{~F}-\mathrm{FDG}$ uptake (a flare in response to estrogen agonists) (17) or a decline in early response to antagonists (18). Recent results from the NeoALTTO trial showed a similar early predictive value for HER2-directed therapy (9). These results support the increasing use of ${ }^{18} \mathrm{~F}$-FDG PET as both an integrated and an integral marker in trials of targeted therapy; however, welldesigned prospective studies are needed to better establish this role for ${ }^{18} \mathrm{~F}-\mathrm{FDG}$ PET.

Molecular imaging probes beyond ${ }^{18} \mathrm{~F}-\mathrm{FDG}$ may be even more sensitive and robust indicators of early drug response. Following the analogy of tissue-based assay of cellular proliferation as earlyresponse indicators, trials of ${ }^{18} \mathrm{~F}$-fluorothymidine PET have shown its value in assessing response as early as $1 \mathrm{wk}$ after initial treatment (Fig. 3) (19). ${ }^{18}$ F-fluorothymidine PET is being tested as an earlyresponse indicator in multicenter trials, including ACRIN 6688, a trial of early breast cancer response to neoadjuvant chemotherapy

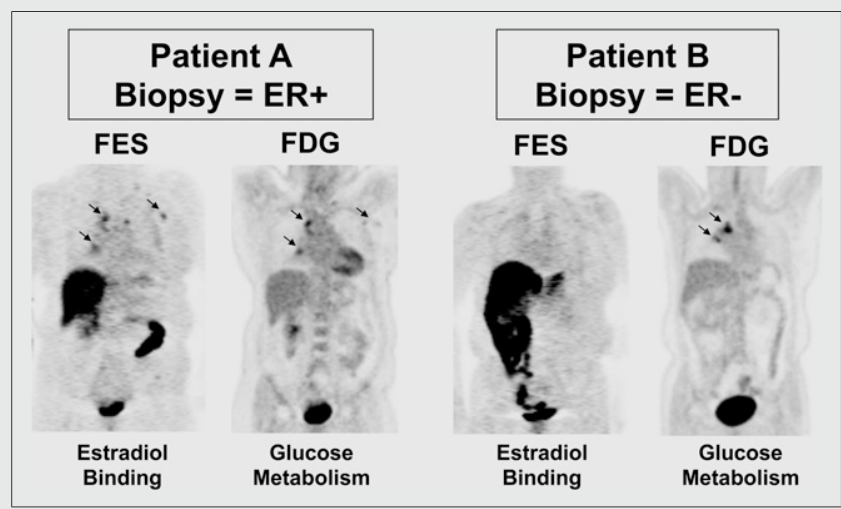

FIGURE 2. Images of ER expression using ${ }^{18} \mathrm{~F}$-fluoroestradiol (FES) PET as example of predictive molecular imaging marker. ${ }^{18} \mathrm{~F}$-fluoroestradiol and ${ }^{18} \mathrm{~F}-\mathrm{FDG}$ PET images are shown from 2 patients, one in whom ${ }^{18} \mathrm{~F}$-fluoroestradiol confirms ER expression (left) and another in whom imaging demonstrates that recurrent tumor has lost ER expression (right). (Reprinted with permission of (14).) performed by the American College of Radiology Imaging Network (ACRIN) under an NCI IND for ${ }^{18} \mathrm{~F}$-fluorothymidine (20). This trial has completed accrual, and results are expected within the next year.

\section{Molecular Imaging as a Surrogate Endpoint}

Imaging is frequently used as a surrogate endpoint for therapeutic trials, most commonly based on changes in tumor size assessed from anatomic imaging according to standard criteria such as the Response Evaluation Criteria in Solid Tumors (RECIST) (21). Although size-based surrogate response endpoints have had success and are commonly used in clinical trials, they have several limitations, including the inability to distinguish residual tumor from nontumor residual tissue masses and significant challenges for assessing response to cytostatic agents when tumor cell death and reduction in tumor mass is not expected. Molecular imaging is beginning to be used in surrogate endpoint criteria-for

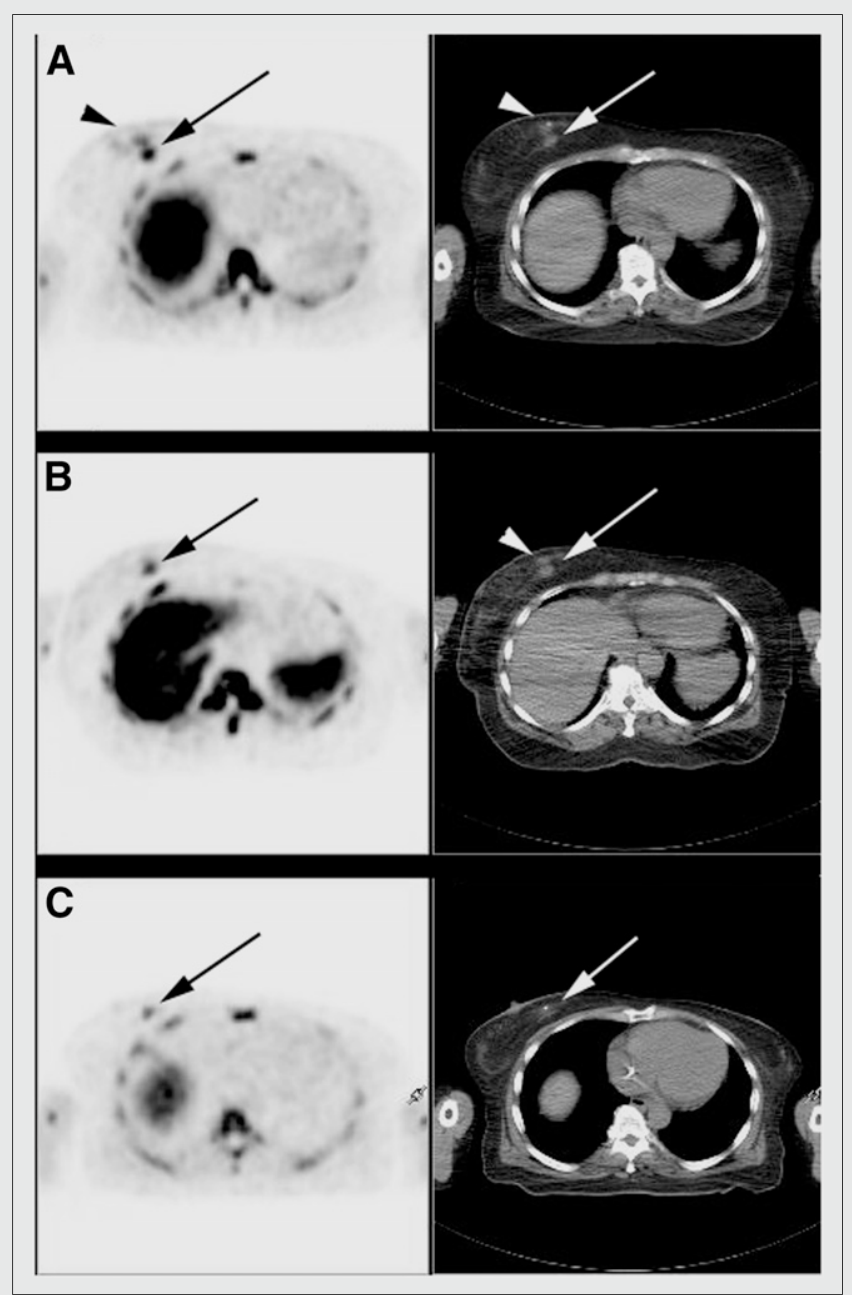

FIGURE 3. ${ }^{18} \mathrm{~F}$-fluorothymidine PET/CT studies at baseline (A), within 1 wk of completion of first cycle of neoadjuvant chemotherapy (B), and after completion of 6 cycles of neoadjuvant chemotherapy and before mastectomy (C). Baseline images show biopsy-proven multifocal disease in right breast on both PET and CT (arrows and arrowheads). After single cycle of chemotherapy, uptake has qualitatively decreased on PET but remains on CT (arrowhead), whereas residual nodule remains on both PET and CT (arrows). After completion of neoadjuvant therapy, only residual nodule re-mains, though it is less intense. Pathology revealed residual $1.7-\mathrm{cm}$ focus of invasive carcinoma with complete response elsewhere in breast. 
example, as an adjunct for identifying disease progression in RECIST 1.1 -though it is not widely accepted as a surrogate endpoint for treatment outcome by regulatory agencies. This fact motivates ongoing efforts to establish molecular imaging, especially ${ }^{18}$ F-FDG PET, as an accepted response endpoint for cancer therapy trials. These efforts have been most successful in lymphoma, for which numerous studies have supported the value of negative posttherapy PET results in predicting survival, and for which ${ }^{18} \mathrm{~F}$-FDG PET is commonly used as an endpoint in clinical trials (22). Although single-center trials have supported the ability of ${ }^{18} \mathrm{~F}$-FDG PET to predict survival in other tumors, including breast cancer $(23,24)$, further prospective studies are needed to support more widespread acceptance of molecular imaging as a surrogate response endpoint. Molecular imaging may be especially helpful in some clinical settings, such as bone metastasis response, in which anatomic imaging is ineffective and early data support the effectiveness of PET as a response endpoint (25).

\section{FUTURE DIRECTIONS}

The development of new predictive and early-response molecular imaging biomarkers using probes designed to quantify specific therapeutic targets or specific pharmacodynamic responses will provide an increasing array of tools to assess drug efficacy as integrated markers in early therapeutic trials. These same markers used as integral biomarkers for patient or therapy selection will increase the utility of phase 2 and 3 drug trials (6). Further studies examining ${ }^{18} \mathrm{~F}$-FDG PET and other molecular imaging tests as robust predictors of patient survival would support the use of molecular imaging as an accepted endpoint in later-stage therapy trials. To achieve these goals, the molecular imaging community must continue to support research and development of new molecular imaging techniques. The molecular imaging community must also come together, working with cooperative clinical trial groups and the pharmaceutical industry, to support well-designed prospective trials validating molecular imaging biomarkers as integrated markers in therapeutic trials, which will lead to later use as integral biomarkers in therapeutic clinical trials and clinical practice.

\section{DISCLOSURE}

No potential conflict of interest relevant to this article was reported.

\section{REFERENCES}

1. Henry NL, Hayes DF. Cancer biomarkers. Mol Oncol. 2012;6:140-146.

2. Hammond ME, Hayes DF, Dowsett M, et al. American Society of Clinical Oncology/College of American Pathologists guideline recommendations for immunohistochemical testing of estrogen and progesterone receptors in breast cancer. J Clin Oncol. 2010;28:2784-2795.

3. McShane LM, Hayes DF. Publication of tumor marker research results: the necessity for complete and transparent reporting. J Clin Oncol. 2012;30:4223-4232.
4. Sargent DJ, Rubinstein L, Schwartz L, et al. Validation of novel imaging methodologies for use as cancer clinical trial end-points. Eur J Cancer. 2009;45: 290-299.

5. Carlson JJ, Roth JA. The impact of the Oncotype Dx breast cancer assay in clinical practice: a systematic review and meta-analysis. Breast Cancer Res Treat. 2013;141:13-22.

6. Shankar LK, Van den Abbeele A, Yap J, Benjamin R, Scheutze S, Fitzgerald TJ. Considerations for the use of imaging tools for phase II treatment trials in oncology. Clin Cancer Res. 2009;15:1891-1897.

7. Kostakoglu L, Gallamini A. Interim ${ }^{18} \mathrm{~F}$-FDG PET in Hodgkin lymphoma: would PET-adapted clinical trials lead to a paradigm shift? J Nucl Med. 2013;54:1082-1093.

8. Kluetz PG, Pierce W, Maher VE, et al. Radium Ra 223 dichloride injection: U.S. Food and Drug Administration drug approval summary. Clin Cancer Res. 2014;20:9-14.

9. Gebhart G, Gamez C, Holmes E, et al. ${ }^{18}$ F-FDG PET/CT for early prediction of response to neoadjuvant lapatinib, trastuzumab, and their combination in HER2positive breast cancer: results from Neo-ALTTO. J Nucl Med. 2013;54:18621868.

10. Dowsett M, Nielsen TO, A'Hern R, et al. Assessment of Ki67 in breast cancer: recommendations from the International Ki67 in Breast Cancer Working Group. J Natl Cancer Inst. 2011;103:1656-1664.

11. DeMichele A, Berry DA, Zujewski J, et al. Developing safety criteria for introducing new agents into neoadjuvant trials. Clin Cancer Res. 2013;19: 2817-2823.

12. Linden HM, Dehdashti F. Novel methods and tracers for breast cancer imaging. Semin Nucl Med. 2013;43:324-329.

13. Tsujikawa T, Yoshida Y, Kudo T, et al. Functional images reflect aggressiveness of endometrial carcinoma: estrogen receptor expression combined with ${ }^{18} \mathrm{~F}$-FDG PET. J Nucl Med. 2009;50:1598-1604.

14. Peterson LM, Kurland BF, Schubert EK, et al. A Phase 2 Study of $16 \alpha-\left[{ }^{18} \mathrm{~F}\right]-$ fluoro-17 $\beta$-estradiol positron emission tomography (FES-PET) as a marker of hormone sensitivity in metastatic breast cancer (MBC). Mol Imaging Biol. October 30, 2013 [Epub ahead of print].

15. Kenny LM, Al-Nahhas A, Aboagye EO. Novel PET biomarkers for breast cancer imaging. Nucl Med Commun. 2011;32:333-335.

16. Weber WA. Assessing tumor response to therapy. J Nucl Med. 2009;50(suppl 1):1S-10S.

17. Dehdashti F, Mortimer JE, Trinkaus K, et al. PET-based estradiol challenge as a predictive biomarker of response to endocrine therapy in women with estrogenreceptor-positive breast cancer. Breast Cancer Res Treat. 2009;113:509-517.

18. Kurland BF, Gadi VK, Specht JM, et al. Feasibility study of FDG PET as an indicator of early response to aromatase inhibitors and trastuzumab in a heterogeneous group of breast cancer patients. EJNMMI Res. 2012;2:34.

19. Contractor KB, Kenny LM, Stebbing J, et al. $\left[{ }^{18} \mathrm{~F}\right]-3^{\prime}$ deoxy- $3^{\prime}$-fluorothymidine positron emission tomography and breast cancer response to docetaxel. Clin Cancer Res. 2011;17:7664-7672.

20. Jolles PR, Kostakoglu L, Bear HD, et al. ACRIN 6688 phase II study of fluorine18 3'-deoxy-3 fluorothymidine (FLT) in invasive breast cancer [abstract]. J Clin Oncol. 2012;29(suppl):TPS125.

21. Eisenhauer EA, Therasse P, Bogaerts J, et al. New response evaluation criteria in solid tumours: revised RECIST guideline (version 1.1). Eur J Cancer. 2009;45: 228-247.

22. Kostakoglu L, Cheson BD. Lymphomas: role of molecular imaging for staging, prognostic evaluation, and treatment response. Front Oncol. 2013;3:212.

23. Dunnwald LK, Doot RK, Specht JM, et al. PET tumor metabolism in locally advanced breast cancer patients undergoing neoadjuvant chemotherapy: value of static versus kinetic measures of fluorodeoxyglucose uptake. Clin Cancer Res. 2011;17:2400-2409.

24. Emmering J, Krak NC, Van der Hoeven JJ, et al. Preoperative [ ${ }^{18}$ F] FDG-PET after chemotherapy in locally advanced breast cancer: prognostic value as compared with histopathology. Ann Oncol. 2008;19:1573-1577.

25. Specht JM, Mankoff DA. Advances in molecular imaging for breast cancer detection and characterization. Breast Cancer Res. 2012;14:206. 